\title{
Bioanalysis
}

\section{What more can TOF-SIMS bring than other MS imaging methods?}

\author{
“...spatial resolution can go down to $2 \mu \mathrm{m}$ for routine SIMS analysis..."
}

\section{First draft submitted: 17 December 2015; Accepted for publication: 13 January 2016; Published online: 19 February 2016}

Keywords: $3 \mathrm{D}$-imaging $\bullet$ DESI $\bullet$ MALDI $\bullet$ MS imaging $\bullet$ sample preparation $\bullet$ secondary ion MS $\bullet$ spatial resolution $\bullet$ structural analysis $\bullet$ reproducibility

MS imaging (MSI) has already become a very popular analytical tool for visualizing the local chemical composition on surfaces and to determine the distribution of each ion detected on the collected mass spectra. Four main methods are now used for this purpose: MALDI [1], focused ion beams (secondary ion MS, SIMS) [2], DESI [3] and inductively coupled plasma MS (ICPMS) [4]. Whereas SIMS imaging was first described in the $60 \mathrm{~s}$, it is often considered as a physicist tool to scrutinize inorganic surfaces. On the contrary MALDI is the most popular as many analytical chemistry laboratories are equipped with MALDI TOF mass spectrometers. Because the DESI is operated at atmospheric pressure, great expectation was put into this technique, but it remains confined to laboratories with a good expertise in technical instrumentation, despite its commercialization for many years. ICP-MS is a very well-known technique for elemental analysis, but this approach is also restrained to a small community and will not be considered here. With the development of cluster ion sources $\left(\mathrm{Au}_{\mathrm{n}}{ }^{+}, \mathrm{Bi}_{\mathrm{n}}{ }^{+}\right.$with $\mathrm{n}<5, \mathrm{C}_{60}{ }^{+}$and $\mathrm{Ar}_{\mathrm{n}}{ }^{+}$with $n>250$ ), TOF-SIMS is regaining of interest especially for the detection of small biomolecules. It is thus interesting to shortly compare the capability of each MSI technique toward TOF-SIMS to understand why the potential of the latter is still largely underestimated.

\section{Spatial resolution}

The main approach for MSI is based on energy deposition onto the analyzed surface, thus inducing desorption and ionization of characteristic ions from it. In that case, spatial resolution is mostly limited by the focalization of the energy source which can be either a primary ion beam under low or atmospheric pressure (SIMS vs DESI) or a laser (MALDI and ICP). MALDI and ICP are therefore limited to the diffraction limit. As Nd:YAG (Nd-dope yttrium aluminum garnet) with tripled frequency or $\mathrm{N}_{2}$ lasers are mostly used for ablation, best attainable spatial resolution is about $700 \mathrm{~nm}$. As far as we know, best performance on a commercial MALDI instrument is about $10 \mu \mathrm{m}$, whereas the team of B. Spengler has developed a homemade MALDI source with ultimate resolution around $2 \mu \mathrm{m}$ [5]. As ions emitted at atmospheric pressure by electrospray are difficult to focus, best spatial resolution for DESI is around $100 \mu \mathrm{m}$. One way to decrease it is to generate primary ions from a nano-electrospray source but this is quite hard to handle for routine analysis. Finally, as primary ions emitted in vacuum can be easily focused by electrostatic lenses, spatial resolution can go down to $2 \mu \mathrm{m}$ for routine SIMS analysis with a mass resolution of a few thousands, down to a few hundreds of $\mathrm{nm}$ if sacrificing mass resolution for biomolecule analysis, and down to tens of $\mathrm{nm}$ for elemental composition (nano-SIMS). Very

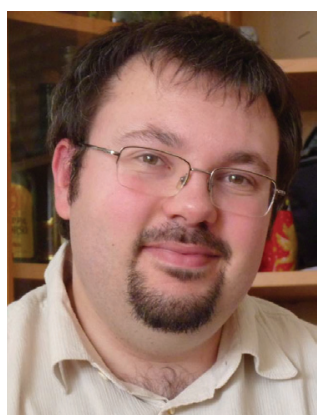

David Touboul Author for correspondence: Institut de Chimie des Substances Naturelles, CNRS UPR 2301, Université Paris-Saclay, Avenue de la Terrasse 91198, Gif-sur-Yvette, France david.touboul@cnrs.fr

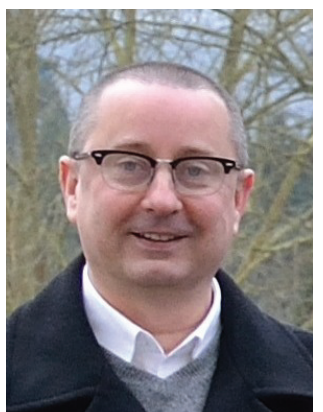

Alain Brunelle

Institut de Chimie des Substances Naturelles, CNRS UPR 2301, Université Paris-Saclay, Avenue de la Terrasse 91198, Gif-sur-Yvette, France 
recently our team demonstrated that delay extraction of secondary ions allow TOF-SIMS image acquisition with a spatial resolution of $400 \mathrm{~nm}$ and an increased mass resolution of about 10,000 using a commercial instrument [6]. As previously stated, such a spatial resolution will never be accessible to MSI based on UV laser ablation indicating the superiority of TOF-SIMS regarding this criterion.

\section{"...TOF-SIMS should probably be considered as the most robust technique for MSI."}

The corollary of spatial resolution is the sensitivity issue. In fact, to decrease the spatial resolution is meaning to decrease the amount of ablated material and thus the sensitivity. If we consider that the depth of ion emission is more or less the same whatever the deposited energy, the sensitivity is decreasing with the square of the spatial resolution. As ionization yields (number of ions over total number of desorbed species - ion plus neutrals) for MALDI or SIMS in the $10^{-4}$ to $10^{-3}$ range have been reported, it is easy to understand why only highly abundant biomolecules, such as lipids from cell membranes, can be detected for spatial resolution below ten microns.

\section{Sample preparation}

Sample preparation is always a key to get reliable information from biosamples. All MSI experiments require flat samples but only MALDI needs a chemical modification of the surface prior analysis. Even if MALDI MSI is probably the most versatile tool in terms of chemical diversity, the sample treatment (buffer wash, delipidation, trypsin digest, matrix choice) is often long and greatly affects the type of detected biomolecules. In fact it is mostly impossible to detect all biochemical classes (polar metabolites, lipids, peptides and proteins) in a single run. On the contrary, DESI and SIMS do not require any sample preparation. Due the desorption/ionization process, the instrument geometry and transmission, applications of these two techniques are principally focused on lipids and other small metabolites which are highly abundant and can be easily ionized. Whereas MALDI or DESI require flat samples to be deposited on a conductive material, insulating samples can be analyzed by SIMS. Examples are very small size samples, like section of Arabidopsis thaliana seeds or cultural heritage artifacts, which usually need to be resin-embedded. In that case, ultramicrotomy is used to remove some sections of less than one micrometer thickness from the sample surface and the images are acquired from the residual block showing a flat and clean surface [7]. If required the block can be 'repol- ished' with minor sample consumption to reanalyze the sample.

\section{Reproducibility}

Reproducibility is crucial when validating analytical methods. Surprisingly very few MSI studies reported standard deviation of peak intensity from region of interest (ROI) on adjacent tissue sections or between groups (treated vs untreated, control vs diseased). For MALDI MSI, the question of measuring reproducibility was mainly addressed by teams working on quantification of exogenous drugs in tissue sections. The most critical step is again the matrix coating which modulates the crystal shape and size, the phenomenon of solvent/matrix extraction occurring at the surface and finally the ionization efficiency. Reasonable reproducibility below $20 \%$ was achieved in few cases but reports on a complete method validation remain scarce. As sample preparation is very limited for TOF-SIMS, better robustness is expected compared with MALDI. We recently published a complete study on this particular point by comparing ion emission and lipid images recorded on right and left hemisphere from the same rat brain section, between three adjacent sections, and from four rats. Instrumental variability was estimated below $15 \%$ and intraand interindividual reproducibility was better than $15 \%[8]$. In that context, TOF-SIMS should probably be considered as the most robust technique for MSI.

\section{Structural analysis}

For peak identification, MS/MS is required. As MALDI sources are coupled with either TOF-TOF or ion trap/quadripole-Orbitrap analyzers, structural information can be obtained on small metabolites, drugs, lipids, peptides or even small proteins. DESI is often hyphenated with ion trap/quadripole-orbitrap analyzers allowing mass assignment using both high mass resolution and MS/MS experiments. In this field one must recognize that SIMS is relatively immature as soon as only homemade tandem instruments are currently available. A promising commercial instrument is coupling a three electrostatic sector TOF analyzer to a MS/MS analyzer fitted with a high energy collision cell. One of the most promising equipment is probably the one developed through a consortium managed by the National Physical Laboratory in UK where a TOFSIMS is coupled to a Q-Orbitrap. The TOF analyzer is used for fast image acquisition at high lateral resolution whereas the Q-Orbitrap analyzes is mandatory for MS and MS/MS data generation with high mass resolution and accuracy. The future commercial release of this instrument is highly expected by the biological TOF-SIMS community. 


\section{D imaging}

Two approaches can be described. The first one requires adjacent tissue sections and successive acquisition of images of each section. In that case with MALDI- or DESI-IMS, tens to hundreds of gigabytes of data are acquired and need to be treated in order to reconstruct $3 \mathrm{D}$ images of each ion of interest. For that purpose, new computational pipelines were developed including alignment with other biological images, segmentation to reduce data size and complexity and efficient visualization tools [9]. By the use of a dual beam approach, TOF-SIMS allows recording of 3D-images on a unique sample with a limited acquisition time of several hours. In that case, a first polyatomic ion beam, such as $\mathrm{C}_{60}{ }^{+}$ or large argon clusters, is used for sputtering and a second one, such as $\mathrm{Bi}_{n}{ }^{+}$clusters, is employed for imaging at a micrometer scale [10]. The main limitation is the restricted imaged areas of about $500 \times 500 \mu \mathrm{m}^{2}$ but 3D-TOF-SIMS offers much more details and a better depth resolution of few tens of nanometers.

\section{References}

1 Norris JL, Caprioli RM. Analysis of tissue specimens by matrix-assisted laser desorption/ionization imaging mass spectrometry in biological and clinical research. Chem. Rev. 113(4), 2309-2342 (2013).

2 Bich C, Touboul D, Brunelle A. Cluster TOF-SIMS imaging as a tool for micrometric histology of lipids in tissue. Mass Spectrom. Rev. 33(6), 4424-4451 (2014).

3 Wu C, Dill AL, Eberlin LS, Cooks RG, Ifa DR. Mass spectrometry imaging under ambient conditions. Mass Spectrom. Rev. 32(3), 218-243 (2013).

4 Sussulini A, Becker JS, Becker JS. Laser ablation ICP-MS: application in biomedical research. Mass Spectrom. Rev. doi:10.1002/mas.21481 (2015) (Epub ahead of print).

5 Römpp A, Schäfer KC, Guenther S, Spengler B et al. Highresolution atmospheric pressure infrared laser desorption/ ionization mass spectrometry imaging of biological tissue. Anal. Bioanal. Chem. 405(22), 6959-6968 (2013).

\section{Conclusion}

Even if MALDI remains the most popular and sensitive technique for MSI, TOF-SIMS offers unique advantages, like high spatial resolution (down to 400 $\mathrm{nm}$ ), sample preparation not limited by the matrix deposition, excellent reproducibility allowing semiquantitative data analysis, and fast $3 \mathrm{D}$ imaging. Nevertheless one of its major limitations is still the lack of mass spectrometers for structural analysis of desorbed ions compared with MALDI or DESI.

\section{Financial \& competing interests disclosure}

The authors have no relevant affiliations or financial involvement with any organization or entity with a financial interest in or financial conflict with the subject matter or materials discussed in the manuscript. This includes employment, consultancies, honoraria, stock ownership or options, expert testimony, grants or patents received or pending, or royalties.

No writing assistance was utilized in the production of this manuscript.

6 Vanbellingen QP, Elie N, Eller MJ, Della-Negra S, Touboul D, Brunelle A. Time-of-flight secondary ion mass spectrometry imaging of biological samples with delayed extraction for high mass and high spatial resolutions. Rapid Commun. Mass Spectrom. 29(13), 1187-1195 (2015).

7 Seyer A, Einhorn J, Brunelle A, Laprévote O. Localization of flavonoids in seeds by cluster time-of-flight secondary ion mass spectrometry imaging. Anal. Chem. 82(6), 2326-2333 (2010).

8 Bich C, Touboul D, Brunelle A. Study of experimental variability in TOF-SIMS mass spectrometry imaging of biological samples. Int. J. Mass Spectrom. 337, 43-49 (2013).

9 Oetjen J, Aichler M, Trede D et al. MRI-compatible pipeline for three-dimensional MALDI imaging mass spectrometry using PAXgene fixation. J. Proteomics 90, 52-60 (2013).

10 Bich C, Havelund R, Moellers R et al. Argon cluster ion source evaluation on lipid standards and rat brain tissue samples. Anal. Chem. 85(16), 7745-7752 (2013). 